\title{
ESCOLHA DE PARCEIROS HETEROSSEXUAIS: UM ESTUDO DE PROCLAMAS DE CASAMENTO
}

\author{
Emma Otta ${ }^{1}$ \\ Renato da Silva Queiroz ${ }^{2}$ \\ Lucila de Sousa Campos ${ }^{3}$ \\ Monika Weronika Dowbor da Silva ${ }^{4}$ \\ Mariana Telles Silveira ${ }^{5}$
}

\begin{abstract}
RESUMO
Este artigo analisa, de uma perspectiva evolucionária e interdisciplinar, critérios de escolha de parceiros heterossexuais com base no fator idade. A pesquisa em que se fundamenta focaliza um total de 3.000 proclamas de casamento publicados no jornal Diário de Notícias de São Paulo, em setembro e outubro de 1996. A publicação de proclamas é um requisito formal para a legalização de uma união civil. Os homens, de
\end{abstract}

\footnotetext{
Professor assistente doutor do Departamento de Psicologia Experimental do Instituto de Psicologia da Universidade de São Paulo.

2 Professor titular do Departamento de Antropologia da Faculdade de Filosofia, Letras e Ciências Humanas da Universidade de São Paulo.

3 Bolsista de Aperfeiçoamento da FAPESP junto ao Departamento de Psicologia Experimental do Instituto de Psicologia da Universidade de São Paulo.

4 Bolsista de Iniciação Científica do CNPq junto ao Departamento de Antropologia da Faculdade de Filosofia, Letras e Ciências Humanas da Universidade de São Paulo.

5 Bolsista de Iniciação Científica do CNPq junto ao Departamento de Psicologia Experimental do Instituto de Psicologia da Universidade de São Paulo.
} 
modo geral, preferiram casar-se com parceiras mais novas que eles, aumentando essa diferença de idade à medida que se situavam em faixas etárias mais avançadas. A única exceção ficou por conta daqueles que se casaram muito jovens, com menos de 20 anos, que buscaram parceiras um pouco mais velhas que eles próprios. As mulheres jovens, por sua vez, casaram-se com homens mais velhos, mas esta tendência diminuiu com o aumento da idade, chegando a se inverter nas faixas de idade mais avançada. De forma geral, o padrão de preferência pela idade do parceiro contraria a regra da atração pelo similar, um dos princípios de seleção de parceiros melhor estabelecidos, mas é compatível, grosso modo, com os pressupostos da teoria evolucionária.

Palavras-chave: seleção de parceiros, evolução, estratégias reprodutivas.

\section{ABSTRACT}

In this article preferences for age of marriage partners by heterosexual men and women were investigated, by adopting an evolutionary and interdisciplinary perspective. A study was made based upon 3.000 announcements of forthcoming marriages sampled from the Brazilian newspaper Diário de Notícias de São Paulo, in September and October 1996. These announcements published in newspapers are a legal requirement to marriage. Men tended to get married to younger women and this preference became more pronounced over the life span. There was only one exception to this trend: young men under 20 years old tended to prefer someone older than themselves. Young women married with older men than themselves but this preference became less deep over the life span and even turned into the opposite. Age preferences violate the similarity-attraction rule, one of the strongest general principle of mate selection, but well fit with an evolutionary model.

Key words: mate selection, evolution, reproductive strategies. 
Este artigo apresenta resultados de uma investigação interdisciplinar mais ampla sobre escolha de parceiros heterossexuais. A abordagem teórica aqui adotada reúne contribuições da Psicologia e da Antropologia Social, tendo como ponto focal a perspectiva evolucionária. Adota-se aqui o pressuposto de que os critérios adotados para a escolha de parceiros derivam de diferentes domínios - biopsicológico e sociocultural - da nossa existência. Conscientes ou inconscientes, estes critérios resultam, todavia, do prolongado processo de evolução que nos fez humanos e foram selecionados porque se revelaram adaptativos tendo em vista a nossa estratégia reprodutiva.

A escolha de parceiros heterossexuais tem sido um tema relativamente bem estudado, abundando publicações especializadas sobre esse assunto notadamente na literatura de procedência norteamericana. Psicólogos sociais, antropólogos e sociobiólogos têm demonstrado que casais assemelham-se mais do que seria esperado ao acaso em relação a um grande número de características físicas (tais como altura, cor dos olhos e até circunferência do punho), psicológicas (atratividade, inteligência e atitudes) e sociais (religião e nível socioeconômico), denominando essa tendência homogamia ou assortative mating (Buss, 1985; Rushton, 1989). Nesse contexto, entretanto, a idade do parceiro surge como uma exceção.

Os homens preferem mulheres mais jovens que eles como parceiras românticas/sexuais, enquanto as mulheres preferem parceiros mais velhos ou aproximadamente da mesma idade. Hayes (1995) analisou anúncios para busca de parceiros publicados em jornais americanos, constatando que, com exceção dos mais jovens, com 20 anos de idade ou menos, em todas as outras faixas etárias os homens buscaram parceiras mais novas. Além disso, quanto mais elevada a faixa etária dos homens, ainda mais jovens eram as parceiras procuradas. A diferença média de idade passou de $-0,13$ na faixa de 25 anos para $-3,63$ na faixa de 35 anos, para $-10,63$ na faixa de 55 anos e $-14,13$ na faixa de 65 anos. 
As mulheres, por sua vez, procuraram homens mais velhos, diminuindo esta diferença quando se situavam em faixas etárias mais elevadas. A diferença média passou de $+4,54$ na faixa de 18 anos para $+3,91$ na faixa de 25 anos, $+3,01$ na faixa de 35 anos, $+1,21$ na faixa de 55 anos e $+0,31$ na faixa de 65 anos. Kenrick, Keefe, Brian, Barr e Brown (1995),Kenrick, Keefe, Gabrielidise Cornelius (1996) e Wiederman (1993), trabalhando com outras amostras de anúncios para busca de parceiros, obtiveram essencialmente os mesmos resultados para os homens. No que diz respeito às mulheres, embora os referidos autores não tenham citado o trabalho de Hayes (1995), é possível, contudo, indicar que não encontraram eles o decréscimo apontado pelo próprio Hayes.

O padrão de preferência pela idade do parceiro é compatível com o modelo evolucionário, segundo o qual fatores biológicos limitam a gama de parceiros com os quais uma pessoa pode se reproduzir (Wiederman, 1993; Kenrick et al., 1992, 1995a, b, 1996). O padrão encontrado é o que está associado à maior probabilidade de sucesso reprodutivo. Os homens tendem a buscar parceiras mais novas que eles próprios em função da capacidade reprodutiva delas. Já as mulheres buscam homens capazes de garantir os recursos necessários à manutenção de uma futura prole. De acordo com o modelo evolucionário, dada a natureza das pressões seletivas, estes homens foram em geral os indivíduos mais velhos (Daly e Wilson, 1983). Ademais, ao contrário do que ocorre com a fertilidade feminina, a masculina não sofre decréscimo acentuado com a idade (Menken e Larsen, 1986).

Os psicólogos sociais apresentam uma justificativa sociocultural para o padrão de preferência pela idade do parceiro, baseada na assimetria de poder entre os sexos (Wiederman e Allgeier, 1992). A exclusão das mulheres da estrutura de poder, associada ao fato de serem vistas como objetos de troca, explicaria o fato delas buscarem nos parceiros românticos/sexuais maiores possibilidades de aquisição de recursos e poder. As mulheres tendem a valorizar recursos 
financeiros, status social e poder, encontrados mais freqüentemente em homens mais velhos (Hayes, 1994, Kenrick e Keefe, 1992). Os homens, em contrapartida, valorizam a beleza física da parceira e seu valor como objeto sexual. Assim, a atratividade física, atributo das mulheres jovens, tornou-se um valor social apreciado pelos homens. Homens com parceiras fisicamente atraentes são percebidos de forma muito mais favorável que aqueles cujas parceiras exibem menos atrativos (Bar-Tal e Saxe, 1976; Sigall e Landy, 1973).

É preciso apontar, contudo, que a maioria destes estudos baseia-se em anúncios para a busca de parceiros, fundamentandose, portanto, na idealização das pessoas. É possível que a realidade seja bastante diferente da expectativa. Assim, um homem poderia desejar uma mulher 20 anos mais nova que ele, mas terminar se casando de fato com uma mulher cinco anos mais velha.

Kenrick e Keefe (1992) estudaram 1.189 registros de casamentos civis realizados em 1986 em duas cidades norte-americanas - Seattle e Phoenix. No caso dos homens, constataram um decréscimo significativo da idade da esposa com o aumento da idade do marido, confirmando, portanto, essencialmente os resultados obtidos com anúncios classificados. No caso das mulheres, verificaram que estas se casaram com homens ligeiramente mais velhos, mantendo-se este padrão constante com o aumento da idade. Observe-se que o trabalho de Kenrick e Keefe (1992) é anterior ao de Hayes (1995, com base em anúncios classificados). Mesmo assim, percebe-se que, no que se refere às mulheres, os resultados encontrados em ambos os artigos são discrepantes.

Um trabalho similar, com base em estatísticas americanas de casamento, foi realizado por Paterson e Pettijohn (1982), cujos resultados mostram que a expressiva maioria dos homens (75,3\%), por ocasião do primeiro casamento, uniu-se a mulheres mais novas; em apenas $9,1 \%$ dos casamentos as mulheres eram mais velhas e em $15,6 \%$ os cônjuges tinham a mesma idade. Esses resultados são congruentes 
com a teoria sociobiológica. No entanto, tal estudo baseou-se numa amostra reduzida ( $\mathrm{N}=275)$, desconsiderando ainda o extenso período de tempo investigado (cinqüenta anos), tendo a discussão ficado restrita apenas à teoria sociobiológica. A propósito, a importância de diferenças socioculturais é sugerida por Buss $(1989,1994)$, num trabalho em que examinou, em 37 culturas, a partir de auto-relatos, a preferência de homens e mulheres pela idade do parceiro. Na Zâmbia e na Colômbia os homens relataram preferir parceiras bem mais jovens do que eles, enquanto nos Estados Unidos essa diferença foi muito menor, situando-se a Polônia e a Itália em posição intermediária. Segundo Glenn (1989), a diferença de idade entre parceiros tende a ser mais pronunciada nos países menos desenvolvidos. Melhores condições de alimentação e saúde, técnicas contraceptivas, somadas ao acesso a recursos estéticos poderiam beneficiar as mulheres dos países desenvolvidos, levando-as a aparentarem uma idade inferior à que têm de fato.

Ademais, convém lembrar que a expectativa de vida nesses países é substancialmente superior à dos países atrasados. A escolarização prolongada, as dificuldades de inserção num mercado de trabalho exigente e competitivo, as relações cada vez mais simétricas entre homens e mulheres e o avanço da idade média das pessoas que se casam e procriam, tudo isso deve exercer alguma influência, no universo dos países mais afluentes, sobre os critérios de escolha de parceiros, notadamente no que diz respeito ao fator idade.

Considerando a já assinalada diversidade que se evidencia quando são confrontados diferentes países, bem como aquela que pode ser identificada no interior de uma única sociedade global, e a ação de fatores decorrentes de peculiaridades socioculturais, julgamos ser estratégica a realização de uma pesquisa levada a efeito a partir de dados pertinentes à realidade brasileira, num momento histórico em que o País vincula-se cada vez mais ao contexto internacional e sofre as influências da assim chamada "modernidade". 
No presente artigo serão examinados 3.000 proclamas de casamento procedentes de cartórios do município de São Paulo, restringindo-se nosso objetivo a discutir a variável idade na formação de casais com base nos laços formais do matrimônio.

\section{MÉTODO}

Foram analisados 3.000 proclamas de casamento publicados no jornal paulistano Diário de Notícias de São Paulo, nos meses de setembro e outubro de 1996. Além da área central do município de São Paulo, escolhemos aleatoriamente bairros situados em cada uma das suas outras quatro zonas geográficas: sul, norte, leste e oeste (bairros: Consolação, Santa Cecília, Cerqueira César, Tucuruvi, Vila Nova Cachoeira, Pirituba, Perdizes, Butantã, Itaquera, Belenzinho, São Miguel Paulista, Santo Amaro, Vila Mariana e Ibirapuera.). Os proclamas analisados provêm, portanto, de cartórios de registro civil localizados em todos esses bairros.

Em metade dos proclamas focalizou-se o homem e em metade, a mulher. Este critério de divisão foi aplicado aos dados pertinentes a cada cartório. Por exemplo: em relação ao cartório do distrito de Perdizes, $50 \%$ dos proclamas foram tabulados focalizando-se a mulher como sujeito e em $50 \%$ o homem.

\section{RESULTADOS}

\section{Idade ao casar}

As mulheres ( $X=25,3 \pm 6,9$ anos) casaram-se com idade significativamente menor que os homens $(X=28,1, \pm 8,0, t=10,36$, $p<0,001$ ).

Quando se dividiu a idade dos sujeitos por faixas etárias (tabela 1), a diferença de gênero ficou especialmente clara. A quantidade de InterAÇÃO, Curitiba, v. 2, p. 97-112, jan./dez. 1998 
mulheres casando-se na faixa de 15-19 anos foi cinco vezes maior que a de homens. Houve inclusive uma mulher casando-se com menos de 15 anos, mas nenhum homem neste caso. Na faixa de 20-24 anos a diferença diminuiu, embora ainda favorecendo as mulheres. $\mathrm{Na}$ faixa de 25-29 anos a diferença se inverteu e os homens passaram a se casar em quantidade superior às mulheres. Daí em diante a diferença a favor dos homens se acentuou.

\section{Diferença entre a idade do parceiro e a idade do sujeito}

Analisou-se a seguir a diferença entre a idade do parceiro e a do sujeito, utilizando-se o mesmo critério de Hayes (1994: 128). Uma diferença positiva mostra que o parceiro é mais velho que o sujeito e uma diferença negativa, que é mais novo.

Constatou-se que $65,8 \%$ dos homens casaram-se com mulheres mais jovens que eles próprios, sendo que a diferença variou de -33 anos a -1 ano (tabela 2). Houve, no entanto, um acúmulo de casos em que a diferença situou-se entre -6 anos e -1 ano (46,7\% do total). Na faixa de diferença zero concentraram-se $10,7 \%$ dos casos. Os demais $23,5 \%$ dos homens casaram-se com mulheres mais velhas que eles, podendo a diferença variar de $+1 \mathrm{a}+44$ anos. A maior parte dos casos, no entanto, situou-se na faixa de +1 a +6 anos (19,6\% do total). 
TABELA 1: DISTRIBUIÇÃO DAS IDADES DOS SUJEITOS NAS AMOSTRAS FEMININA E MASCULINA.

\begin{tabular}{|c|c|c|c|c|}
\cline { 2 - 5 } \multicolumn{1}{c|}{} & \multicolumn{2}{c|}{ Homens } & \multicolumn{2}{c|}{ Mulheres } \\
\hline Classes de Idade & $\mathrm{n}$ & $\%$ & $\mathrm{n}$ & $\%$ \\
\hline$<15$ & 0 & 0 & 1 & 0,1 \\
\hline $15-19$ & 51 & 3,4 & 250 & 16,7 \\
\hline $20-24$ & 499 & 33,3 & 573 & 38,2 \\
\hline $25-29$ & 503 & 33,5 & 378 & 25,2 \\
\hline $30-34$ & 240 & 16 & 159 & 10,6 \\
\hline $35-39$ & 101 & 6,7 & 77 & 5,1 \\
\hline $40-44$ & 42 & 2,8 & 33 & 2,2 \\
\hline $45-49$ & 19 & 1,3 & 16 & 1,1 \\
\hline $50-54$ & 11 & 0,7 & 5 & 0,3 \\
\hline $55-59$ & 16 & 1,1 & 4 & 0,3 \\
\hline $60-64$ & 7 & 0,5 & 1 & 0,1 \\
\hline$<65$ & 11 & 0,7 & 3 & 0,2 \\
\hline
\end{tabular}

TABELA 2: DISTRIBUIÇÃO DAS DIFERENÇAS DE IDADE ENTRE $O$ PARCEIRO E O SUJEITO NAS AMOSTRAS FEMININA E MASCULINA.

\begin{tabular}{|l|c|c|c|c|}
\cline { 2 - 5 } \multicolumn{1}{c|}{} & \multicolumn{4}{c|}{ Sexo do sujeito } \\
\cline { 2 - 5 } \multicolumn{1}{c|}{ Parceiro } & $\mathrm{n}$ & $\%$ & $\mathrm{n}$ & $\%$ \\
\hline Mais novo & 987 & 65,8 & 357 & 23,8 \\
\hline Até 6 anos de diferença & 700 & 46,7 & 298 & 19,9 \\
\hline 7 anos de diferença ou mais & 287 & 19,1 & 59 & 3,9 \\
\hline Mesma idade & 161 & 10,7 & 152 & 10,1 \\
\hline Mais velho & 352 & 23,5 & 991 & 66,1 \\
\hline Até 6 anos de diferença & 294 & 19,6 & 709 & 47,3 \\
\hline 7 anos de diferença ou mais & 58 & 3,9 & 282 & 18,8 \\
\hline
\end{tabular}


A distribuição das diferenças de idade entre o parceiro e o sujeito para a amostra feminina espelha a distribuição masculina, 66,1\% das mulheres casaram-se com homens mais velhos, estendendo-se a diferença de +1 a +39 anos, com um acúmulo de casos na faixa de +1 a +6 anos de diferença $(47,3 \%$ do total). Na faixa de diferença zero situaram-se $10,1 \%$ dos casos. No restante $23,8 \%$ das mulheres casaram-se com homens mais novos que elas, variando a diferença de -25 a -1 ano. A maior parte dos casos, no entanto, situou-se na faixa de -6 a -1 ano (19,9\%).

A figura 1 mostra a diferença média entre a idade do parceiro e a do sujeito em função da idade do sujeito, para as amostras feminina e masculina. Como se pode verificar, predomina aí o padrão de preferência dos homens pelas mulheres mais jovens. Os dados revelam uma tendência bastante regular, os homens preferindo mulheres cada vez mais jovens à medida que eles próprios se tornam mais velhos. A única exceção ficou por conta dos homens que se casaram muito jovens, com menos de vinte anos, que buscaram parceiras um pouco mais velhas que eles próprios. As mulheres, por sua vez, apresentaram um padrão diferente. As de menos de 30 anos casaram-se com homens mais velhos que elas. Quanto mais jovem a mulher, maior a diferença de idade em relação ao marido. Com o aumento da idade a diferença diminuía, até que na faixa de 30-45 anos a diferença tornava-se praticamente desprezível. Finalmente, na faixa de 45 anos ou mais encontramos uma tendência das mulheres casarem-se com homens mais jovens que elas próprias. 


\section{FIGURA 1: DISTRIBUIÇÃO DAS DIFERENÇAS DE IDADE ENTRE O PARCEIRO E O SUJEITO EM FUNÇÃO DA IDADE E SEXO DO SUJEITO.}

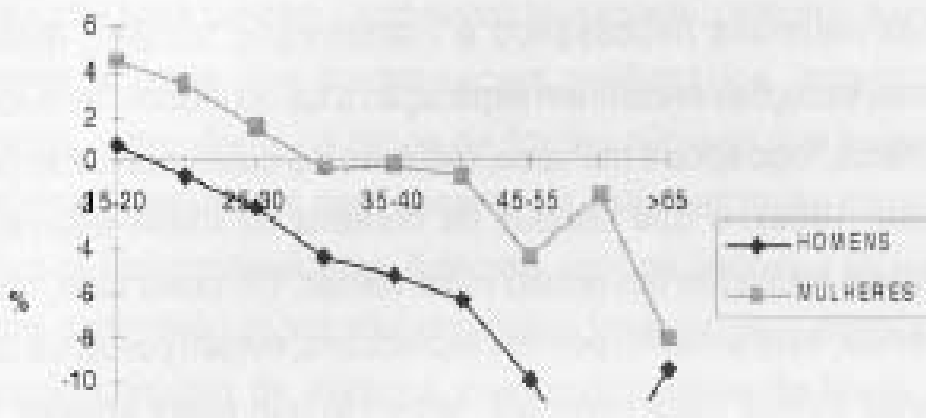

\section{DISCUSSÃO}

Os resultados encontrados no presente estudo são consistentes com os relatados na literatura especializada que se valeu de diferentes tipos de fontes: auto-relato (Buss, 1989), anúncios classificados de procura de parceiros (Hayes, 1995; Kenrick et al., 1995a, b, 1996; Wiederman, 1993) e proclamas de casamento (Patersone Pettijohn, 1982). Os homens revelaram uma preferência por mulheres mais jovens, exceção feita àqueles situados em faixas etárias inferiores, que revelaram certa preferência por mulheres um pouco mais velhas. Por sua vez, as mulheres casaram-se de início com homens mais velhos, decrescendo essa tendência com o aumento da idade, passando a casarem-se então com homens de idade equivalente às suas e, por fim, com homens mais novos. Esse padrão de preferência por idade é compatível com o modelo evolucionário, segundo o qual a biologia estabelece limitações na gama de parceiros com os quais uma pessoa pode se reproduzir.

A preferência encontrada nos dados em análise é a prevista pela maior probabilidade de sucesso reprodutivo. Convém observar que o 
amadurecimento sexual das mulheres ocorre mais cedo, o que as leva a procurarem parceiros numa faixa etária mais avançada, fisicamente compatível com elas (Paterson e Pettijohn, 1982) e que já acumularam recursos materiais necessários à manutenção familiar. Mesmo as aparentes exceções encontram explicação à luz do modelo evolucionário. As mulheres, logo após a menarca, exibem uma subfecundidade (Menken e Larsen, 1986), o que conduz os homens de idade equivalente a procurarem mulheres um pouco mais velhas. De outro lado, mulheres mais velhas, mas ainda no período reprodutivo, evitam parceiros situados nas faixas etárias mais elevadas, temendo que estes possam falecer ainda durante a infância dos filhos.

A tendência de serem os homens representados em maior número nas faixas etárias mais elevadas pode ser explicada também pela biologia da reprodução, já que seu potencial reprodutivo declina muito mais lentamente ao longo do tempo, enquanto a capacidade reprodutiva da mulher decresce mais rapidamente com o avançar da idade, justificando-se assim a menor presença feminina nos proclamas de casamento na faixa de 45 anos em diante.

Como se sabe, a fertilidade feminina atinge um pico na faixa etária de 20-30 anos, mantendo-se elevada até os 35 anos, aproximadamente, declinando, então, sobretudo a partir da faixa dos 40 anos. Em torno dos 50 anos, com a menopausa, as mulheres perdem a capacidade biológica da procriação (Menken e Larsen, 1986). Ademais, em torno dos 40 anos cresce a probabilidade de má formação do feto (como as mulheres já nascem com todos os óvulos, estes vão envelhecendo à medida que o tempo passa, enquanto os homens produzem espermatozóides diariamente) e de gravidez de alto risco, aumentando também a taxa de mortalidade perinatal e complicações outras para a saúde materna (Resnik, 1986).

Esta tendência também poderia ser explicada por meio de um enfoque sociocultural. A expectativa social é de que a mulher ou 
viúva mantenha-se voltada para a família - filhos, netos e trabalho doméstico, ficando sob maior controle familiar. Todavia, os homens nesta situação civil não sofrem a mesma pressão, podendo construir mais facilmente uma nova relação. Lembramos igualmente o estigma que atinge os homens adultos que permanecem celibatários, sendo muito comentados, a propósito, os casos de figuras públicas que teriam feito uniões formais justamente para escapar da estigmatização inerente às acusações de homossexualismo. Ademais, embora parte das sociedades os homens dependem do trabalho doméstico feminino para terem acesso a um amplo conjunto de serviços que tradicionalmente ficam sob a responsabilidade das mulheres (Davis, 1990). Pode-se visualizar essa questão com maior clareza no contexto das sociedades indígenas, nas quais o homem adulto solteiro vê-se em situação difícil, e às vezes até mesmo deplorável, já que lhe é vedado o acesso a um rol expressivo de bens e serviços atribuídos culturalmente apenas e tão-somente ao trabalho feminino (Clastres, 1974; Lévi-Strauss, 1949).

A expectativa social quanto à sexualidade dos dois sexos é assimétrica. Enquanto ao homem é dado o pleno direito ao prazer sexual, a mulher é mais reprimida na sua sexualidade. Casando-se ao final da sua idade reprodutiva, a mulher afirmaria uma sexualidade dissociada da reprodução, fato normalmente visto com reservas à luz da normas estabelecidas.A união entre uma mulher madura e um homem bem mais jovem, por exemplo, sempre desperta a atenção pública e dá margem a comentários jocosos.

É preciso considerar, contudo, o fato de que os proclamas de casamento, que constituem a base da presente investigação, refletem apenas uma parcela das uniões efetivadas. Neste sentido, pode-se indagar até que ponto as uniões não formalizadas reproduziriam as tendências observadas nos proclamas. Outra questão interessante diz respeito às mulheres que optam pela chamada "produção independente". Será que o comportamento delas segue o que seria previsto pelo padrão evolutivo? 
Uma discussão em torno da escolha, pela mulher, do pai de seu filho surgiu recentemente no caso de Madonna, que diz ter escolhido um tipo específico de homem para ser o pai da criança que teve há pouco tempo. No Brasil, a apresentadora de TV Xuxa tem anunciado a intenção de proceder de maneira idêntica. Até que ponto existiria liberdade de escolha em qualquer direção independentemente do padrão evolutivo?

Alguns caminhos de pesquisas futuras não são desprovidos de interesse à luz de reflexões sugeridas pelo presente trabalho, a saber: a) análise de padrões temporais, comparando-se proclamas de casamento em décadas sucessivas desde o início do século 20; b) comparação de proclamas de casamento inicialmente no interior de uma mesma região, focalizando diferenças socioeconômicas para posteriormente se comparar diferentes regiões geográficas, observandose diferenças culturais e sua influência na escolha do parceiro. Espera-se encontrar uma atenuação da diferença de idade entre os sujeitos homens e suas parceiras nas sucessivas faixas etárias, comparando-se o início do século com o momento atual e diferentes segmentos de nível socioeconômico, pressupondo-se que nas camadas mais abastadas haveria uma alteração similar. 


\section{REFERÊNCIAS BIBLIOGRÁFICAS}

BAR-TAL, D.; SAXE, L. Perceptions of similarly and dissimilarly attractive couples and individuals. Journal of Personality and Social Psychology, n. 33, p. 772-781, 1976.

BUSS, D. M. Human mate selection. American Scientist, n. 73, p. 47-51, 1985.

Sex difference in human mate preferences: evolutionary hypotheses tested in 37 cultures. Behavioral and Brain Sciences, n. 12, p. 1-49, 1989.

. The strategies of human mating. American Scientist, n. 82, p. 238249, 1994.

CIASTRES, P. La société contre l'état: recherches d'antropologie politique. Paris: Les Éditions de Munuit, 1974.

DALY, M.; WILSON. Sex, evolution and behavior. Boston: Willard Grand Press, 1983.

DAVIS, S. Men as success objects and women as sex objects: a study of personal advertisements. Sex Roles, n. 23, p. 43-50, 1990.

GIENN, N. D. Intersocietal variation in the mate preferences of males and females. Behavioral and Brain Sciences, n. 12, p.21-23, 1989.

HAYES, A. F. Age preferences for same-and opposite-sex partners. The Journal Social Psychology, v. 135, n. 2, p. 125-133, 1994.

KENRICK, D. T.; KEEFE, R. C. Age preferences in mates reflect sex differences in human reproductive strategies. Behavioral and Brain Sciences, n. 15, p. 75-133, 1992.

Age preferences and mate choice among homosexuals and heterosexuals: a case for modular psychological mechanisms. Journal Personality and Social Psychology, v. 69, n. 6, p. 1.166-1.172, 1995.

; BRYAN, A.; BARR, A.; BROWN, S. Age preferences and mate choice among homosexual and heterosexuals: a case for modular psycological mechanisms. Journal Personality and Social Psychology, n. 69 , p. $1.166-1.172,1995$. 
; __ ; GABRIELIDIS, C.; CORNELIUS, J. S. Adolescents age preferences for dating partners: support for an evolutionary model of Life. History Strategies. Child Development, n. 67, p. 1.499-1.511, 1996.

LÉVI-STRAUSS, C. Les structures élémentaires de la parenté. Paris: PUF, 1949.

MENKEN, J. ; LARSEN, U. Fertility rates and aging. In: MASTROIANNI JR, L.; PAULSEN, C. A. (Eds.). Aging, reproduction, and the climacteric. New York: Plenum Press, 1986.

PATERSON, C.; PETTIJOHN, T. F. Age and human mate selection. Psychological Reports, n. 51, p. 70, 1982.

RESNIK, R. Age related changes in gestation and pregnancy outcome. In: MASTROIANNI JR, L.; PAULSEN, C. A. (Eds.). Aging, reproduction and the climacteric. Nova York: Plenum Press, 1986.

RUSHTON, J. P. Genetic similarity, human altruism, and group selection. Behavioral and Brain Sciences, n. 12, p. 503-545, 1989.

SIGALL, H.; LANDY, D. Radiating beauty: effects of having a physically attractive partner on person perception. Journal of Personality and Social Psychology, n. 28, p. 219-224, 1973.

WIEDERMAN, M. W. Evolved gender differences in mate preferences: evidence from personal advertisements. Ethology and Sociobiology, n. 14, p. 331- 352, 1993.

; ALLGEIER, E. R. Gender differences in mate selection criteria: sociobiological or socioeconomic explanation? Ethology and Sociobiology, n. 13, p. 115-124, 1992. 